\title{
Percepções de pacientes sobre alimentação no seu processo de adoecimento crônico por síndrome metabólica: um estudo qualitativo
}

\author{
Perceptions of patients with metabolic syndrome \\ regarding their diet during onset of the \\ disease: a qualitative study
}

Carla Maria VIEIRA ${ }^{1}$

Egberto Ribeiro TURATO'

RE S U M O

Objetivo

Investigar aspectos psicoculturais que envolvem as práticas alimentares e a imagem corporal no processo de adoecimento crônico por síndrome metabólica.

\section{Métodos}

Estudo clínico-qualitativo realizado por meio de entrevistas semidirigidas de questões abertas, submetidas à técnica de análise de conteúdo e interpretação dos dados com base no referencial teórico psicodinâmico. Foram entrevistados nove indivíduos em tratamento ambulatorial para síndrome metabólica em hospital universitário em Campinas (SP).

\section{Resultados}

Os resultados foram organizados empiricamente em três categorias: "Quem é gordo é horrível"; "O peso é muito e a gordura estorva demais"; "Como muito pouco. Ninguém acredita". Os sujeitos do estudo revelaram rejeição à obesidade, reforçada pela cultura de enaltecimento da magreza. A insatisfação com a imagem corporal leva os pacientes a um desvio de foco do problema, o que interfere no desenvolvimento do autocuidado e no manejo do tratamento dos distúrbios que caracterizam a síndrome metabólica. Tanto a dissociação entre as práticas alimentares e o excesso de peso quanto a utilização dos alimentos para preencher lacunas de ordem emocional expressam dificuldades de enfrentamento dos conflitos, o que colabora com o desvio de foco acima referido.

\section{Conclusão}

O cuidado nutricional e as propostas dietéticas para portadores de síndrome metabólica devem estabelecer escuta acurada das demandas emocionais, que se manifestam por meio da insatisfação com a imagem

${ }^{1}$ Universidade Estadual de Campinas, Faculdade de Ciências Médicas, Departamento de Psicologia Médica e Psiquiatria. Cidade Universitária Zeferino Vaz, Caixa Postal 6111, 13083-970, Campinas, SP, Brasil. Correspondência para/Correspondence to: C.M. VIEIRA. E-mails: <carlmari@fcm.unicamp.br>; <cmvieira@unimep.br>. 
426 | C.M. VIEIRA \& E.R. TURATO

corporal, das dificuldades para estabelecer mudanças nas práticas alimentares e/ou dos episódios de compulsão alimentar.

Termos de indexação: Autocuidado. Fatores psicológicos. Nutrição. Obesidade. Práticas alimentares. Síndrome Metabólica.

\section{A B S T R A C T}

\section{Objective}

This study aimed to investigate the psychological and cultural issues regarding dietary habits and body image during the onset of metabolic syndrome.

\section{Methods}

A clinical, qualitative study was conducted with a sample of nine outpatients being treated for metabolic syndrome at a university teaching hospital in Campinas, São Paulo, Brazil. Data were collected using semistructured interviews with open questions and then submitted to content analysis. Interpretation of data was based on a psychodynamic theoretical framework.

\section{Results}

The results were organized empirically into three categories: "Fat people look terrible"; "I am too heavy and my weight bothers me"; and "I eat so little; nobody believes it". The participants in this study revealed their rejection of obesity, reinforced by a culture that exalts thinness. Dissatisfaction with their body image resulted in the patients deflecting the focus of the problem, thereby affecting the development of self-care and management of the treatment for the metabolic disorders that characterize metabolic syndrome. Dissociation between dietary habits and excess weight, as well as the use of food to fill emotional gaps, reflect difficulties in facing conflicts and contribute towards deflecting the focus of the problem.

\section{Conclusion}

Nutritional care and diets proposed for patients with metabolic syndrome should take into consideration emotional demands resulting from dissatisfaction with body image, difficulties in implementing changes in dietary habits and episodes of compulsive overeating.

Indexing terms: Self-care. Nutrition. Psychological factors. Obesity. Food practices. Metabolic Syndrome.

\section{N T R O D U ÇÃ O}

Considerada um dos problemas de saúde pública emergentes em nossa sociedade, a Síndrome Metabólica (SM) apresenta-se na literatura com amplo reconhecimento da complexidade de fatores envolvidos em sua conceituação, etiologia e tratamento ${ }^{1}$. A caracterização clínica da SM tem sido descrita por meio da associação de pelo menos três dos seguintes sintomas: alteração dos níveis de glicemia, com ou sem diabetes tipo II; gordura abdominal acumulada; dislipidemias; níveis pressóricos elevados; microalbuminúria e outros distúrbios renais que se agravam com a cronicidade do processo de adoecimento ${ }^{2}$.

Esse conjunto de agravos, no entanto, nem sempre é reconhecido como SM. Entidades internacionais que organizam os consensos e estabelecem parâmetros de diagnóstico, tratamento e prevenção no campo de estudos da endocrionolgia e metabologia apresentam divergências conceituais em virtude da complexidade dos fatores causais e das diferentes manifestações clínicas e abordagens terapêuticas, o que dificulta a delimitação epidemiológica do problema $a^{1,3,4}$. Apesar dessa dificuldade, há forte convergência de opiniões descritas na literatura sobre o aumento da prevalência dos sintomas e dos fatores de risco associados a padrões dietéticos do mundo ocidental ${ }^{3,5}$.

Ações preventivas e estímulo ao comportamento de autocuidado são frequentemente indicados como tratamento de primeira linha para portadores de SM ${ }^{4,6,7}$. No entanto, estudos envol- 
vendo questões psicoculturais com pacientes de SM relatam baixa adesão às mudanças de hábitos, sobretudo no que se refere às práticas alimentares e ao estilo de vida ${ }^{8-10}$.

Em relação ao fenômeno alimentar, tem sido discutida a importância da determinação dos aspectos psicoculturais nas práticas alimentares. Ao discutirem a questão da adesão às propostas de mudanças de hábito, essas abordagens reforçam a necessidade de se considerar a subjetividade nas práticas de cuidado nutricional8,9,11,12.

Distúrbios no comportamento alimentar podem vincular-se a processos mentais muito primitivos da infância, com reflexos no desenvolvimento do aparelho psíquico e na formação do pensamento. Essas experiências primitivas que formam a base psicológica infantil podem interferir nas respostas futuras de aceitação dos alimentos e de sua rejeição, ampliadas ao longo da vida pelas redes de relações interpessoais e interações culturais socialmente constituídas ${ }^{13}$.

Do ponto de vista psicodinâmico, o comportamento de compulsão alimentar é também compreendido como uma associação de eventos da vida de uma pessoa em que a mente encontra-se insuficientemente forte para suportar uma dor, um sofrimento psíquico, e descarrega a tensão de origem emocional em atos repetitivos. Nessa perspectiva, o comer por impulso pode ser a manifestação da dificuldade de uma pessoa lidar com um momento de fragilidade, de estafa, de um trauma, ou com histórias de vida em que predominam situações de vulnerabilidade capazes de desencadear mecanismos psíquicos de compulsão no campo alimentar, com efeitos no estado nutricional ${ }^{13}$.

A dificuldade em aceitar e gostar do próprio corpo, entre outros problemas, encontra-se associada ao comportamento alimentar compulsivo. Muitos sujeitos que manifestam o excesso de peso como uma das consequências da compulsividade na maneira de se alimentar recorrem a dietas restritivas com o objetivo de perder peso e melhorar sua imagem corporal. Porém, com o desejo potencializado pelos períodos de carência não satisfeita, eles voltam a engordar sistematicamente ${ }^{14,15}$.

Questões de ordem emocional mesclam-se às influências de origem cultural no campo alimentar, tais como a idealização de um modelo de beleza que se torna inatingível, o que dificulta o desenvolvimento de autoestima e aceitação da imagem corporal. Nesse sentido, estudos socioantropológicos denunciam o surgimento da moral da boa forma como padrão estético hegemônico, uma cultura que exige dos indivíduos o controle de sua aparência física. A rejeição à obesidade é propagada pela mídia através do enaltecimento da magreza, do culto ao corpo jovem e saudável, o que dissemina a ideia de que o corpo gordo não tem saúde ${ }^{16-18}$.

O fenômeno atual de repúdio à obesidade, entre outros aspectos psicoculturais que envolvem as práticas alimentares e a imagem corporal, tem reflexos no manejo das enfermidades metabólicas. A importância do tema é sugerida pelo estudo sobre as vivências e as percepções dos sujeitos relativas ao processo de adoecimento crônico com relações estreitas com o fenômeno alimentar.

A valorização do ponto de vista do paciente sobre a doença oferece contribuições singulares para a ampliação das práticas em saúde e para a formulação das ações e políticas públicas de prevenção e tratamento de agravos metabólicos crônicos vinculados à nutrição. Saberes sobre o manejo das diferentes fases do adoecimento crônico que se determina no contexto sociocultural e na história de vida dos pacientes complementam o conhecimento técnico dos profissionais da saúde e contribuem na condução do processo terapêutico.

Ao desenvolver a escuta acurada sobre as questões psicoculturais que envolvem o fenômeno alimentar, este estudo pretende contribuir para que esses aspectos sejam considerados no âmbito do cuidado, o que representaria melhora no atendimento a essa população. 


\section{MÉTODOS}

O modelo de pesquisa qualitativa em saúde foi aplicado neste estudo, adotando-se uma abordagem humanística na busca de sentidos e significados de experiências pessoais vivenciadas em situações cotidianas ${ }^{19}$. A adoção do método de pesquisa clínico qualitativa lhe confere um caráter exploratório, não experimenta|20.

O método de pesquisa clínico qualitativa se sustenta em três pilares que se caracterizam por: valorizar as angústias e ansiedades humanas, como força motriz para a pesquisa; desenvolver a postura clínica voltada a uma escuta qualificada sobre o sofrimento do indivíduo sob estudo, movida pela atitude de cuidado; e fazer a interação afetiva entrevistador - entrevistado ${ }^{20}$.

Este estudo contou com nove pacientes escolhidos de maneira intencional para compor o grupo de sujeitos ${ }^{19,21}$ (Quadro 1). O critério de inclusão dos sujeitos foram: pacientes adultos em seguimento clínico com diagnóstico de SM, que concordaram explicitamente em participar da investigação, conforme Termo de Consentimento Livre e Esclarecido. O estudo foi aprovado pelo Comitê de Ética em Pesquisa da Faculdade Ciências Médicas da Universidade Estadual de Campinas, (parecer n 809/2005 CAAE 1738.0.146.000-05).

Foram considerados como elementos de identificação/especificação dados sociodemográficos como idade, estado civil, ocupação (Quadro 1). A pesquisa de campo foi realizada em ambulatório para SM, localizado num complexo hospitalar universitário público, na cidade de Campinas, região sudeste do Brasil, durante o período de outubro de 2006 a fevereiro de 2008.

A coleta de dados ocorreu por meio da observação das atividades clínicas ambulatoriais, registradas em diário de campo, da aplicação de entrevistas em profundidade, semidirigidas, com questões abertas, assim como da identificação das manifestações não verbais e sentimentos percebidos nas entrevistas. Trata-se de um processo indutivo que procura atingir, mediante o diálogo, o aprofundamento da entrevista e a interpretação do que não está dito, buscando significados atribuídos ao objeto do estudo em lacunas que se encontram além do que é gravado e transcrito ${ }^{22}$. Para tanto, impressões do pesquisador e anotações em diário de campo foram fundamentais na análise dos dados ${ }^{19}$.

As entrevistas foram gravadas e transcritas em arquivos de texto para posterior aplicação da técnica de leituras e releituras flutuantes, deixando-se impregnar pelo seu conteúdo ${ }^{22}$. Para apreender a subjetividade dos entrevistados foram levados em conta o discurso manifesto e o discurso subentendido nas entrelinhas, nas expressões do olhar, nos gestos, ou seja, na linguagem corporal. O conjunto do material obtido a partir das entrevistas foi submetido à técnica de análise de conteúdo a fim de identificar as categorias empíricas. A categorização por núcleos de sentido foi a técnica adotada para o desenvolvimento da análise qualitativa de conteúdo ${ }^{23}$.

Quadro 1.Caracterização dos sujeitos entrevistados: dados sociodemográficos e nutricionais. Campinas (SP), 2006-2008.

\begin{tabular}{ll}
\hline Sujeitos & Idade; Situação conjugal; Ocupação atual; Tempo registrado de tratamento em prontuário; Estado nutricional \\
\hline A & 48 anos; Casada; Dona de casa; 2 anos de tratamento; Obesidade Grau III \\
B & 63 anos; Casada; Empregada doméstica; 10 anos de tratamento; Sobrepeso com gordura abdominal acumulada \\
C & 45 anos; Casada; Atividade agropecuária; 7 anos de tratamento; Obesidade Grau III \\
D & 48 anos; Casado; Açougueiro licenciado; 4 anos de tratamento; Obesidade Grau III \\
E & 53 anos; Casada; Dona de casa; 6 anos de tratamento; Obesidade grau III \\
F & 37 anos; Casada; Dona de casa; 13 anos de tratamento; Obesidade grau III \\
G & 49 anos; Divorciada; Cozinheira licenciada; 11 anos de tratamento; Obesidade grau III \\
H & 55 anos; Casado; Pedreiro aposentado; 6 anos de tratamento; Obesidade grau II \\
I & 54 anos; Solteira; Auxiliar de enfermagem; 17 anos de tratamento; Sobrepeso após cirurgia bariátrica em 2003 \\
\hline
\end{tabular}


As categorias delineadas no presente estudo foram discutidas e interpretadas com base no referencial teórico psicodinâmico ${ }^{24}$. A abordagem interdisciplinar prevalece neste estudo, na qual conhecimentos das áreas da psicologia, da psicanálise e da socioantropologia são aplicados ao campo da saúde para a compreensão do fenômeno alimentar e questões subjacentes ${ }^{25}$.

\section{RESULTADOS E DISCUSSÃO}

Foram levadas em conta, para a análise, as experiências pessoais de convivência com o processo de adoecimento de sujeitos portadores de SM. Suas respostas foram classificadas em três categorias, que receberam como títulos fragmentos dos discursos dos sujeitos, utilizando metáforas para caracterizar o conteúdo que emergia das análises: 1 - "Quem é gordo é horrível"; 2 - "O peso é muito! E a gordura estorva demais"; 3 - "Eu como muito pouco... ninguém acredita".

\section{Quem é gordo é horrível}

A rejeição à obesidade e uma concepção de que o corpo gordo é um corpo sem saúde foram identificadas a partir da análise dos resultados:

... Eu comecei a ver que eu não estava bem, estava ficando cada vez mais obesa, mais horrivel, eu falo horrivel mesmo, porque quem é gordo é horrivel (I, , , 54 anos, sobrepeso).

A insatisfação em relação ao excesso de peso se fez evidente como uma questão central na sua compreensão sobre o processo de adoecimento:

... não estou aguentando... por causa do peso... (D, O, 48 anos, obesidade III).

... problema de saúde sempre agrava... complicando mais porque fica mais pesado... (E, Q, 53 anos, obesidade III).

$\mathrm{O}$ excesso de peso foi revelado como um pesado fardo emocional a ser carregado em suas vidas. Os sujeitos condensam no corpo gordo toda a problemática e justificam seus conflitos emocionais pela questão do peso. Assim, ao fixar-se na insatisfação com sua imagem corporal, o paciente tende a perder o foco do processo de adoecimento crônico por SM e do desenvolvimento de atitudes de autocuidado.

Não aparece com o mesmo sentido a percepção dos sujeitos quanto à convivência com os outros distúrbios metabólicos que caracterizam a $\mathrm{SM}$, tais como diabetes, hipertensão e dislipidemia. Diferentemente da obesidade, essas doenças não parecem sofrer o mesmo grau de rejeição.

As percepções sobre o excesso de peso são reforçadas pelo fenômeno de rejeição social e cultural à obesidade. A obesidade é visualmente considerada um incômodo, uma característica que não se pode esconder, enquanto que outros distúrbios metabólicos podem passar quase despercebidos nas relações sociais ${ }^{17}$.

Em estudo sobre a cultura de enaltecimento da magreza e preocupação excessiva com a gordura corporal, Sudo \& Madel Luz ${ }^{17}$ identificaram o respaldo dessa cultura pelas mensagens na mídia que colocam o gordo como vilão da sociedade, e que favorecem a opressão sobre um tipo de indivíduo que possui no seu corpo uma marca que o põe em uma condição oposta ao que é considerado ideal. Esse indivíduo não estaria cumprindo um dever, ao não aproveitar os recursos que estão sendo ofertados pela ciência e pelo saber biomédico, onerando a sociedade pelas consequências da obesidade para o sistema de saúde ${ }^{17}$.

Nesse contexto, a pessoa com excesso de peso vivencia estigmas paradoxais. Por um lado é simpática, extrovertida e corresponde ao gosto pela boa mesa e pelo convívio. Por outro lado, suscita reprovação, discriminação social por portar um estereótipo de doente, depressiva e irresponsável. Sem controle sobre si mesma, é considerada portadora de uma característica pessoal de fraqueza diante dos apelos da gula ${ }^{18}$. 
430 | C.M. VIEIRA \& E.R. TURATO

\section{O peso é muito! E a gordura estorva demais}

A percepção dos sujeitos sobre sua gordura corporal nos revela um sentido de desvitalizaçao. O excesso de gordura foi revelado como algo que Ihe rouba saúde e que não Ihe pertence.

E faz muito mal... sinto muito cansaço... O peso é muito! E a gordura... estorva demais... (F, O, 37 anos, obesidade III).

A gordura, simbolizada pelos sujeitos como algo que, além de não Ihes pertencer, atrapalha suas vidas, faz emergir a dificuldade em assumir a responsabilidade pela condição de excesso de peso. Os sujeitos, ao denunciarem a falta de controle sobre seu próprio corpo, revelam também a dificuldade de lidar com suas emoções e seus conflitos.

Se tivesse peso normal não estaria correndo tanto risco assim... (F, O, 37 anos, obesidade III).

Eu pensei que eu tivesse emagrecido... o exame me deixou triste... Comi dois salgados, tomei refrigerante... (G, o, 49 anos, obesidade III).

Foram revelados sentimentos de medo, tristeza e impotência em vista do insucesso na busca pela redução do excesso de peso. Associado a esses sentimentos, os sujeitos manifestaram o desejo de submeter-se às intervenções cirúrgicas indicadas para obesidade severa.

A gente tenta, tenta e não consegue perder o peso... eu fico mais triste é que eu não consigo perder o peso. Mesmo fazendo a dieta... eu deveria perder pelo menos uns 40 quilos... eu faço de tudo (sorriso) ... prá ... fazer a redução do estômago, porque todos os regimes eu fiz mas... nada consegui ( $\mathrm{F}, \mathrm{Q}, 37$ anos, obesidade III).

Nesse contexto, a cirurgia bariátrica significa uma possibilidade de livrar-se da gordura corporal. O significado da cirurgia, porém, como solução "mágica" denuncia a dificuldade dos sujeitos em assumir uma postura ativa no manejo da obesidade assim como dos outros agravos metabólicos.

O desejo de submeter-se à cirurgia para solucionar seus problemas de excesso de peso revela também mais uma tentativa de camuflar seus conflitos de ordem emocional, vinculados à insatisfação com a imagem corporal e à rejeição do corpo gordo.

\section{Como muito pouco... ninguém acredita}

Com o objetivo de explicar o ganho excessivo de peso, os sujeitos não consideram o excesso de gordura corporal como um resultado das suas práticas alimentares.

Eu venho ganhando peso direto... como muito pouco... ninguém acredita... eu me sinto como uma mentirosa... (C, o, 45 anos obesidade III).

A contradição nos discursos sobre o fenômeno alimentar, expressa através de um jogo de palavras "muito" e "pouco", nos revela que o "muito" pode ser "pouco" para satisfazer ou preencher lacunas, que podem ser constatadas nas entrelinhas, nas manifestações não verbais ou no silêncio das entrevistas. Os alimentos, mesmo sendo "muito" e satisfazendo momentaneamente, ainda são insuficientes para aplacar uma fome de ordem emocional, que poderá se manifestar em atos de compulsão alimentar.

Não acharam o problema que eu tenho por não comer... já passei muito mal por ficar sem comer ( $C$, o, 45 anos, obesidade III).

Engordei devido à preocupação. Porque minha filha não está se dando bem também com meu genro (G, O, 49 anos, obesidade III).

Não alimento bem... tomo 30 comprimidos por dia... essa é a alimentação razoável minha... Tenho é raiva... da comida (A, ơ, 48 anos, obesidade III). 
Como os sujeitos que apresentam distúrbios alimentares nem sempre conseguem perceber a fome como uma manifestação fisiológica, o ato de comer acaba cumprindo funções de satisfazer demandas emocionais. Quando o ato de se alimentar se torna exclusivo do desejo, não sendo mais o apetite regulado pela fome, a alimentação pode configurar um pano de fundo de conflitos psíquicos, seja por excesso, seja por rejeição a ela 26,27 .

Bruno $^{13}$, em artigo sobre compulsão, aborda os distúrbios alimentares como uma "articulação da mente que não está suficientemente preparada para enfrentar o real e desenvolve recursos de fuga". Na medida em que o sofrimento psíquico não consegue ser vivenciado conscientemente, chega a provocar quebra do sistema regulador dos afetos, podendo ser canalizado para a ação motora: a compulsão. Portanto, a compulsão ocorreria para ajudar a suportar uma dor de ordem emocional, transformando-a em dor corporal. O corpo vem em socorro da mente através de um agir repetitivo e angustiado para proteger a pessoa de uma dor psíquica que ela não tem condições de resolver ou suportar ${ }^{13}$.

Esses indivíduos nem sempre conseguem perceber que utilizam os alimentos para "satisfazer" demandas emocionais. Dessa forma, o cuidado nutricional e as propostas dietéticas devem considerar as dificuldades dos portadores de SM em adequar suas práticas alimentares.

\section{O N C L U S Ã O}

A insatisfação com o excesso de peso e o sentimento de impotência diante de tentativas fracassadas para emagrecer significaram, tal como relatados pelos sujeitos deste estudo, uma esperada preocupação pessoal quanto às vivências da evolução crônica no adoecimento por SM. Enquanto a percepção desses indivíduos dava relevo ao fenômeno da obesidade, sua linguagem verbal e não verbal naturalmente ressaltavam sua rejeição à gordura corporal.

Mesmo que os pacientes reconheçam a obesidade como problema importante de saúde, nossos resultados sugerem que o manejo adequado, por parte deles, da evolução crônica do adoecimento por distúrbios metabólicos pode estar fortemente dificultado pelo padrão cultural atual de enaltecimento da magreza e pela cultura lipofóbica.

Observamos, nas falas, determinada dissociação entre as práticas alimentares e os determinantes do ganho excessivo de peso, assim como emerge o uso dos alimentos para preencher lacunas de ordem afetiva, revelando dificuldades desses sujeitos em lidar com conflitos emocionais. A falta de elementos psicológicos internos para a elaboração da autonomia e do desenvolvimento da capacidade de tomar a responsabilidade por cuidar de si indica uma inibição das possibilidades de adaptações psicossociais referentes ao campo alimentar, dentre outras dimensões do autocuidado.

Sugerimos que a compreensão sobre os fenômenos alimentares, por parte dos pacientes e dos profissionais, deva ser ampliada para além dos aspectos nutricionais, justificando-se pela crucial importância da boa condição dessa nutrição para a melhora do quadro que compõe a SM, bem como para a prevenção em especial de eventos cardiovasculares. As dificuldades relatadas pelos pacientes para estabelecer mudanças nesse campo de vida confirmaram-se estar associadas aos afetos ambivalentes, mobilizados na sua relação simbólica com a comida e com o próprio corpo.

\section{COLABORADORES}

Este artigo foi elaborado a partir da tese de doutoramento da primeira autora, em andamento, com dados das entrevistas por ela realizadas, com a participação do segundo autor na orientação, análise e discussão dos dados.

REFERÊ N CIAS

1. Alberti KGMM, Zimmet P, Shaw J. Metabolic syndrome: a new world-wide definition. A 
Consensus Statement from the International Diabetes Federation. Diabetes Mellitus. 2006; 23:469-80.

2. Sociedade Brasileira de Hipertensão. I Diretriz brasileira de diagnóstico e tratamento da síndrome metabólica. conceituação, epidemiologia e diagnóstico. Rev Bras Hipertens. 2004; 7(4):130-1.

3. Geloneze B. Síndrome metabólica: mito ou realidade? Arq Bras Endocrinol Metab. 2006; 50(3): 409-11.

4. Opie LH. Metabolic syndrome. Circulation. 2007; 115:e32-e35.

5. Oliveira EP, Souza MLA, Lima MDA. Prevalência de síndrome metabólica em uma área rural do semiárido baiano. Arq Bras Endocrinol Metab. 2006; 50(3):456-65.

6. McLellan KCP, Barbalho SM, Cattalini M, Lerarrio AC. Diabetes Mellitus do tipo 2, síndrome metabólica e modificação no estilo de vida. Rev Nutr. 2007; 20(5):515-24. doi: 10.1590/S1415-52732 007000500007.

7. Santos CRB, Portella ES, Ávila SS, Soares EA. Fatores dietéticos na prevenção e tratamento de comorbidades associadas à síndrome metabólica. Rev Nutr. 2006; 19(3):389-401. doi: 10.1590/S1415-5 2732006000300010.

8. Sharovsky LL, Perez GH, Romano BW, Lopes HF. Psicoterapia de grupo em pacientes portadores de síndrome metabólica. Rev Soc Cardio Estado de São Paulo. 2004; 14:646-51.

9. Perez GH, Romano BW. O comportamento alimentar e síndrome metabólica: aspectos psicológicos. Rev Soc Cardio Estado de São Paulo. 2004; 14(4):652-60.

10. Corica F, Corsonello A, Apolone G, Mannucci E, Lucchetti M, Bonfiglio C, et al. Metabolic syndrome, psychological status and quality of life in obesity: the QUOVADIS study. Int J Obes. 2008; 32(1):185.

11. Viana V. Psicologia, saúde e nutrição: contributo para o estudo do comportamento alimentar. Anál Psicol. 2002; 4:611-24.

12. Toral N, Slater B. Abordagem do modelo transteórico no comportamento alimentar. Ciên Saúde Coletiva. 2007; 12(6):1641-50.

13. Bruno CANB. Com pulsão e distúrbios alimentares. Psique. 2009; 39.

14. Contreras Hernández J. La obesidad: uma perspectiva sociocultural. Zainak. 2005; 27:31-52.

15. Bernardi F, Cichelero C, Vitolo MR. Comportamento de restrição alimentar e obesidade. Rev Nutr. 2005;
18(1):85-93. doi: 10.1590/S1415-5273200500 0100008.

16. Ferreira FR. A produção de sentidos sobre a imagem do corpo. Interface. 2008; 12(26):47-83.

17. Sudo N, Madel Luz T. O gordo em pauta: representações do ser gordo em revistas semanais. Ciên Saúde Coletiva. 2007; 12(4):1033-40.

18. Yoshino NL. O corpo "em excesso" e o culto da "boa forma". In: Canesqui AM, organizador. Olhares socioantropológicos sobre os adoecidos crônicos. São Paulo: Hucitec; 2007. p.111-27.

19. Minayo MCS. Desafio do conhecimento: pesquisa qualitativa em saúde. $8^{a}$ ed. São Paulo: Hucitec; 2004.

20. Turato ER. Tratado da metodologia da pesquisa clínico-qualitativa: construção teórico-epistemológica, discussão comparada e aplicação nas áreas humanas e da saúde. $3^{a}$ ed. Petrópolis: Vozes; 2008.

21. Fontanella BJB, Rica J, Turato ER. Abordagem por saturação em pesquisas qualitativas em saúde: contribuições teóricas. Cad Saúde Pública. 2008; 24(1):17-24.

22. Fontanella BJB, Campos CJG, Turato ER. Coleta de dados na pesquisa clínico-qualitativa: uso de entrevistas não dirigidas de questões abertas por profissionais da saúde. Rev Lat-Am Enfermagem. 2006; 14(5):812-20.

23. Campos CJG, Turato ER. Análise de conteúdo em pesquisas que utilizam metodologia clínico-qualitativa: aplicação e perspectivas. Rev Lat-Am Enfermagem. 2009; 17(2):259-64.

24. Ferraz FC, Volich RM, organizadores. Piscossoma: psicossomática psicoanalítica. São Paulo: Casa do Psicólogo; 1997.

25. Garcia RWD. A antropologia aplicada às diferentes áreas da nutrição. In: Canesqui AM, Garcia RWD, organizadores. Antropologia e nutrição: um diálogo possível. Rio de Janeiro: Fiocruz; 2005. p.287-303. Coleção Antropologia e Saúde.

26. Espíndola CR, Blay SL. Bulimia e transtorno de compulsão alimentar periódica: revisão sistemática e metassíntese. Rev Psiquiatr RS. 2006; 28(3): 265-75.

27. Castillo DC. Apetito y nutricion. Rev Chil Pediatr. 1990; 61(6):346-53.

Recebido em: 7/3/2008

Versão final reapresentada em: 3/5/2010 Aprovado em: 6/5/2010 